\title{
7th Asian Congress for Microcirculation (ACM'08)
}

\author{
Date: October 17-19, 2008 \\ Venue: $\quad$ Ramada Plaza, Taian, Shandong, China \\ Website: www.ACM2008.net
}

Sponsored by: China International Exchange Center,

Chinese Society of Microcirculation (CSM)

Organized by: Taishan Medical University,

Institute of Microcirculation, CAMS \& PUMC

Supported by: China Association of Science and Technology

Affiliated by: The Asian Union for Microcirculation (AUM)

Congress theme: "Microvascular Biology: From Basic to Clinic"

Congress topics (but not limited to): Clinical detection and evaluation; Tumor; Cardiovascular disease; Cerebrovascular diseases; Diabetes mellitus; Organ-specific microcirculation; Lymphatic function; Tissue regenesis; Proteomics; Angiogenesis and regulation; Ischemia and reperfusion injury; Microvascular inflammation and leukocyte function; Stem cell biology; Endothelial cell biology and function; Oxidative stress/free radicals; Imaging evaluation of microcirculation/blood perfusion; Oriental medicine; Artificial blood; Oxygen/blood transport to tissue.

Deadline for abstract: August 25, 2008

Registration fee: US $\$ 300$ - before September 15, 2008

US $\$ 380$ - after September 15, 2008

Secretariat ACM'08: E-mail: acm.taishan@yahoo.com.cn

Fax: +861066251957 (Beijing), +86 53862299532 (Taian) 